\title{
Direct interaction between $S$-locus receptor kinase and M-locus protein kinase involved in Brassica self-incompatibility signaling
}

\author{
Mitsuru Kakita, Hiroko Shimosato, Kohji Murase ${ }^{a}$, Akira Isogai, Seiji Takayama* \\ Graduate School of Biological Sciences, Nara Institute of Science and Technology, Nara 630-0192, Japan \\ *E-mail: takayama@bs.naist.jp Tel: +743-72-5450 Fax: +743-72-5459
}

Received November 22, 2006; accepted December 27, 2006 (Edited by M. Umeda)

\begin{abstract}
Many flowering plants possess self-incompatibility (SI) systems to prevent inbreeding. In Brassica, SI recognition is controlled by the multiallelic gene complex ( $S$-haplotypes) at the $S$-locus, which encodes both the male determinant ( $S$-locus protein 11 (SP11/SCR)) and the female determinant ( $S$-receptor kinase (SRK)). After self-pollination, an $S$-haplotype specific interaction between pollen-borne SP11 and its cognate stigmatic SRK receptor induces SI signaling in stigma papilla cells, which results in the rejection of the self-pollen. Previous genetic analysis by our group of a selfcompatible mutant revealed the involvement of a membrane-anchored cytoplasmic protein kinase, $M$-locus protein kinase (MLPK), in SI signaling. The plasma membrane localization of MLPK suggests that it functions in the vicinity of SRK, but the physiological relationship between the two proteins remains unknown. In the present study, we looked for a direct interaction between SRK and MLPK using both genetic and biochemical techniques. Although a conventional yeast twohybrid system and a split-ubiquitin membrane-based yeast two-hybrid system failed to detect a direct interaction between SRK and MLPK, an in vitro phosphorylation assay indicated that the kinase domain of SRK could efficiently phosphorylate MLPK. These results suggest that MLPK could be a direct target of SRK in Brassica SI signaling.
\end{abstract}

Key words: Brassica rapa, phosphorylation, receptor-like cytoplasmic kinase, receptor kinase, self-incompatibility.

Self-incompatibility (SI) is a genetic system in flowering plants that promotes outbreeding by preventing selfpollination (Takayama and Isogai 2005). SI is based on self/nonself discrimination processes between pollen and pistil, followed by selective inhibition of the germination and/or growth of self-pollen. In crucifers, SI recognition is controlled by a multiallelic gene complex (termed the $S$-haplotypes; $S_{1}, S_{2}, \ldots, S_{\mathrm{n}}$ ) at a single locus, termed the $S$-locus. More than one hundred $S$-haplotypes are inferred to be present in Brassica rapa (Nou et al. 1993).

Genomic analyses of the $S$-locus in crucifers revealed the presence of two multiallelic genes: $S$-receptor kinase $(S R K)$ (Stein et al. 1991) and S-locus protein 11 (SP11, also called $S$-locus cysteine rich: $S C R$ ) (Suzuki et al. 1999; Takayama et al. 2000). SRK encodes a membranespanning serine/threonine receptor kinase that localizes to the papilla cell membrane and functions as the sole determinant of the stigma SI phenotype (Takasaki et al. 2000). SP11 encodes a small cysteine-rich protein that localizes to the pollen coat and functions as the sole determinant of the SI phenotype of pollen (Schopfer et al. 1999; Takayama et al. 2000; Shiba et al. 2001). Biochemical studies have clearly shown that SP11 is a ligand for SRK, and the specificity of the SI response can now be explained by the $S$-haplotype-specific interactions between the SP11 ligand and the SRK receptor (Takayama et al. 2001). During self-pollination, the pollen-borne SP11 ligand specifically binds to the extracellular domain of its cognate self SRK and activates its kinase domain (Cabrillac et al. 2001; Takayama et al. 2001). This activation apparently triggers a signaling cascade that results in the inhibition of pollen hydration, germination, and pollen tube penetration into the papilla cell wall. However, our understanding of the signal transduction pathway downstream of SRK is limited.

Arm repeat containing 1 (ARC1), a stigmatic U-box protein with E3 ubiquitin ligase activity, is a potent positive mediator of this signal transduction pathway (Stone et al. 2003). ARC1 interacts with the kinase domain of SRK in a phosphorylation-dependent manner via a C-terminal arm repeat domain, and its antisense suppression resulted in a partial breakdown in the SI response (Stone et al. 1999). In one model, ARC1 is activated by SRK and then promotes the ubiquitination and proteasomal degradation of unknown "compatibility factors" in the pistil, which in turn leads to pollen rejection. However, most parts of this model still require

${ }^{\text {a }}$ Present address: Department of Biology, LSRC B327, Box 91000, Duke University, Durham, NC 27708-1000, USA

Abbreviations: ARC1, arm repeat containing-1; MLPK, $M$-locus protein kinase; SP11/SCR; S-locus protein 11/S-locus cysteine rich; SRK, $S$-receptor kinase.

This article can be found at http://www.jspcmb.jp/ 
experimental validation.

We recently identified another positive mediator of SI signaling by genetically analyzing a spontaneous selfcompatible mutant of $B$. rapa var. yellow sarson (Murase et al. 2004). Yellow sarson has a recessive mutation of the modifier $(\mathrm{m})$ gene, which completely eliminates the stigma SI response. Positional cloning of the $M$ gene revealed that it encodes a cytoplasmic serine/threonine protein kinase, designated $M$ locus protein kinase (MLPK). The mutant form of mlpk has a missense mutation in the conserved kinase subdomain VIa, which results in the loss of kinase activity. MLPK belongs to a subfamily of the receptor-like cytoplasmic kinases (RLCKs), which share a common monophyletic origin with receptor-like kinases but have no apparent signal sequences or transmembrane domains (Shiu and Bleecker 2001). Although the genome of Arabidopsis thaliana contains as many as 150 RLCKs, very little is known about their function. MLPK is the only example demonstrating the mediation of an RLCK member in the signaling of receptor-like kinases.

MLPK has a common plant myristoylation consensus sequence, M-G-X\{not EDFKRVWY\}-X-X-[STACFGRV]$\mathrm{X}\{$ not DE\}, at its N-terminus (Boisson et al. 2003) and is localized to the stigmatic plasma membrane (Murase et al. 2004). The plasma membrane localization of MLPK suggests that it may function in the vicinity of SRK, but the physiological relationship between SRK and MLPK has not yet been elucidated. In the present study, we examined the possible direct interaction between SRK and MLPK using both genetic and biochemical systems.

\section{Materials and methods}

\section{Entry vector constructions}

Total RNA was isolated from the stigmas of Brassica rapa $S_{8} S_{8}$ and $S_{9} S_{9}$ homozygotes one day prior to anthesis using ISOGEN (Nippon gene, Toyama, Japan) according to the manufacturer's instructions. First strand complementary DNA (cDNA) was synthesized from $1 \mu \mathrm{g}$ of DNase I-treated total RNA using Superscript II RNaseH-Reverse Transcriptase (Invitrogen, Carlsbad, CA). The following forward primers were used to PCR amplify the cDNA: for MLPK, MLPKF (5'-ATGGGGATTTGCATGAGTGTTCAG- $\left.3^{\prime}\right)$ and MLPKR (5'-TCAGACAAACAGAGGCGAAGCAGACGGAC-3'); for $S R K_{8} K D$, SRK8KDF (5'-CAAAATCGAGCAAAAGCAATGGCA-3') and SRK8R (5'-TCCGCGGTTACCGGGCATCGATGACTGAG-3'); for fulllength $S R K_{8}$, SRK8F (5'-ATGCAAGGTGTACGATACATCTATC-3') and SRK8R2 (5'-CCGGGCATCGATGACTGAGCAGGT-3'); for $S R K_{9} K D$, SRK9KDF (5'-CAAAGGCGAGCAAAAGCAATGGCA-3') and SRK9R (5'-AACCGCGGTTAGCGGGCATCTATGACTGAG-3'); and for full-length $S R K_{9}$, SRK9F (5'-ATGAAAGGTGTACGAAACAT-3') and SRK9R2 (5'-GCGGGCATCTATGACTGAGCAGGT-3'). The amplified fragments were cloned into the $\mathrm{pCR}^{\circledR} 8 / \mathrm{GW} / \mathrm{TOPO}$ cloning vector (Invitrogen) and sequenced.

To generate the kinase-deficient mutant of $M L P K^{K 112 R}$, the lysine at position 112 was mutated to arginine in the $M L P K$ entry vector using the PCR-based GeneTailor SiteDirected Mutagenesis System (Invitrogen) according to the manufacturer's instructions. The following two primers were used: MLPK-K112R-1 (5'-GGCATGGTTATTGCTGTCAGAAGACTTAACATA-3') and MLPK-K112R-2 (5'-GACAGCAATAACCATGCCGGTTCCCGGTTT).

\section{Yeast two-hybrid assay}

Matchmaker Two-Hybrid System 3 (Clontech, Palo Alto, CA) was used for the yeast two-hybrid assay. The bait and prey vectors, pGBKT7 and pGADT7 (Clontech), were converted to the Gateway-compatible destination vectors, $\mathrm{pGBK} / \mathrm{gw}$ and pGAD/gw, respectively, using the Gateway vector conversion system (Invitrogen). The entry vectors (constructed as above) were recombined into the $\mathrm{pGBK} / \mathrm{gw}$ and $\mathrm{pGAD} / \mathrm{gw}$ vectors using Gateway LR Clonase (Invitrogen).

Yeast cells of strain AH109 were co-transformed with the bait and prey vectors ( $\mathrm{pGBK} / \mathrm{gw}$ and $\mathrm{pGAD} / \mathrm{gw}$ derivatives) using a lithium acetate-based protocol and grown on synthetic dextrose media. Transformants were first spread on SD/-Leu/Trp plates, and then co-transformed positive colonies were grown on $\mathrm{SD} /$-Leu/-Trp/-Ade/-His plates to detect activation of the HIS3 and $A D E 2$ reporter genes. A prey vector containing SV-40 large T-antigen (pGAD-T) and a bait vector containing murine p53 (pGBKT7-53) were used as a positive control system (Clontech). A bait vector containing human lamin $\mathrm{C}$ (pGBKT7-Lam) was used as a negative control (Clontech).

\section{Split-ubiquitin membrane-based yeast two- hybrid assay}

DUALsystem 3 (Dualsystems Biotech, Zurich, Switzerland) was used for the split-ubiquitin membrane-based yeast twohybrid assay. The bait and prey vectors pCCW-SUC and pPR3C (Dualsystems Biotech), respectively, were converted to the Gateway-compatible destination vectors $\mathrm{pCCW}-\mathrm{SUC} / \mathrm{gw}$ and pPR3-C/gw, respectively, using the Gateway vector conversion system (Invitrogen). The entry vectors (constructed as above) were recombined into $\mathrm{pCCW}-\mathrm{SUC} / \mathrm{gw}$ and $\mathrm{pPR} 3-\mathrm{C} / \mathrm{gw}$ vectors using Gateway LR Clonase mix (Invitrogen).

Yeast cells of strain NMY32 were co-transformed with the bait and prey vectors (pCCW-SUC/gw and pPR3-C/gw derivatives) using a lithium acetate-based protocol and grown on synthetic dextrose media. Transformants were first spread on SD/-Leu/-Trp plates, and then co-transformed positive colonies were grown on $\mathrm{SD} /$-Leu/-Trp/-Ade/-His plates to detect activation of the HIS3 and $A D E 2$ reporter genes. Expression and membrane localization of the SRK and MLPK fusions were confirmed by Western blot analyses using an antiLexA antibody (Santa Cruz Technologies, Santa Cruz, CA) after introduction into yeast cells (strain DSY-5) as pCCW-SUC constructs. A prey vector expressing Alg5-NubI (wild type $\mathrm{Nub}$ ) and a bait vector expressing Alg5-Cub-LexA-VP16 were used as a positive control (Dualsystems Biotech). A prey vector expressing Alg5-NubG (mutated Nub) and a bait vector expressing Alg5-Cub-LexA-VP16 were used as a negative control. 


\section{Preparation of recombinant SRK kinase domain and MLPK}

To express recombinant proteins as fusions of glutathione S-transferase (GST) and maltose-binding protein (MBP), we used the pGEX5x-3 (Amersham Bioscience, Piscataway, NJ) and pMAL-c2E (New England Biolabs, Beverly, MA) vectors, respectively. These vectors were converted to Gatewaycompatible destination vectors (pGEX/gw and $\mathrm{pMAL} / \mathrm{gw}$ ) using the Gateway vector conversion system (Invitrogen). $S R K_{8} K D$ and $M L P K^{K 112 R}$ entry vectors (constructed as above) were recombined into $\mathrm{pGEX} / \mathrm{gw}$ and $\mathrm{pMAL} / \mathrm{gw}$, respectively. For the production of MBP (control) protein, intact pMAL-c2E vector was used. The vectors were introduced into Escherichia coli BL21-codon plus (DE3)-RIL (Stratagene, Heidelberg, Germany). The transformed E. coli cells were cultured in $500 \mathrm{ml}$ of $2 \times$ YTA medium containing $50 \mu \mathrm{g} \mathrm{ml}^{-1}$ ampicillin at $37^{\circ} \mathrm{C}$. When the culture reached an $\mathrm{OD}_{600}$ of 0.8 , isopropyl $\beta$ D-thiogalactoside was added to a final concentration of $0.1 \mathrm{mM}$ and the culture was incubated at $37^{\circ} \mathrm{C}$ for an additional $3 \mathrm{~h}$.

To purify the GST-SRK ${ }_{8} \mathrm{KD}$ protein, E. coli collected from $500 \mathrm{ml}$ cultures were resuspended in $20 \mathrm{ml}$ of lysis-G buffer (50 mM Tris- $\mathrm{HCl}, 150 \mathrm{mM} \mathrm{NaCl}, 1 \mathrm{mM}$ EDTA, 0.5\% NP-40, $1 \mathrm{mM}$ DTT, and protease inhibitor cocktail (Roche, Basel, Switzerland), $\mathrm{pH} 7.5$ ), sonicated, and centrifuged at $16,000 \times \mathrm{g}$ for $30 \mathrm{~min}$. The supernatant was incubated for $1 \mathrm{~h}$ at $4^{\circ} \mathrm{C}$ with $1 \mathrm{ml}$ of Glutathione Uniflow Resin (Clontech). The resin was collected in a column and washed first with $10 \mathrm{ml}$ TBS buffer (50 mM Tris- $\mathrm{HCl}, 150 \mathrm{mM} \mathrm{NaCl}, \mathrm{pH} 7.5$ ) containing $3 \mathrm{M}$ $\mathrm{NaCl}$, and second with $10 \mathrm{ml}$ TBS. Bound proteins were eluted in TBS containing $20 \mathrm{mM}$ reduced glutathione and dialyzed with reaction buffer $(50 \mathrm{mM}$ Hepes- $\mathrm{KOH}, 100 \mathrm{mM} \mathrm{NaCl}$, $5 \mathrm{mM} \mathrm{MgCl}_{2}, 5 \mathrm{mM} \mathrm{MnCl} 2,1 \mathrm{mM}$ DTT, $0.05 \%$ (w/v) Triton X$100, \mathrm{pH} 7.5)$ containing $10 \%(\mathrm{v} / \mathrm{v})$ glycerol for use in the subsequent kinase assay.

To purify the MBP and MBP-MLPK ${ }^{\mathrm{K} 112 \mathrm{R}}$ proteins, E. coli collected from $500 \mathrm{ml}$ cultures were resuspended in $20 \mathrm{ml}$ of lysis-M buffer $(20 \mathrm{mM}$ Tris- $\mathrm{HCl}, 100 \mathrm{mM} \mathrm{NaCl}, 5 \mathrm{mM} \beta$ mercaptoethanol, protease inhibitor cocktail (Roche), $\mathrm{pH}$ 7.4), sonicated, and centrifuged at $16,000 \times g$ for $30 \mathrm{~min}$. The supernatant was incubated for $1 \mathrm{~h}$ at $4^{\circ} \mathrm{C}$ with $1 \mathrm{ml}$ of amylose resin (New England Biotech). The resin was collected in a column and washed with $10 \mathrm{ml}$ lysis-M buffer containing $1 \mathrm{mM}$ EDTA. Bound proteins were eluted with lysis-M buffer containing $10 \mathrm{mM}$ maltose and dialyzed with reaction buffer containing $10 \%(\mathrm{v} / \mathrm{v})$ glycerol for use in the subsequent kinase assay.

\section{In vitro phosphorylation assay}

For the in vitro phosphorylation assay, approximately $0.5 \mu \mathrm{g}$ of each recombinant protein $\left(\mathrm{GST}-\mathrm{SRK}_{8} \mathrm{KD}, \mathrm{MBP}-\mathrm{MLPK}^{\mathrm{K} 112 \mathrm{R}}\right.$, and MBP), purified as above, was mixed in $10 \mu \mathrm{l}$ reaction buffer containing $50 \mu \mathrm{M}$ ATP and $10 \mu \mathrm{Ci}\left[\gamma^{32} \mathrm{P}\right]$-ATP (GE Healthcare, Piscataway, NJ) and incubated for $15 \mathrm{~min}$ at room temperature. The proteins were separated on a $10 \%$ SDS-PAGE gel. The gel was stained with Coomassie brilliant blue and then dried and processed to detect phosphoproteins using a BAS2500 IP Reader (Fujifilm, Tokyo, Japan).

\section{Results}

To detect a possible direct interaction between SRK and MLPK, we first tested the yeast two-hybrid system, a conventional genetic system used to analyze proteinprotein interactions in vitro. Because this system is only suitable for soluble proteins, we used only the kinase domains of the SRKs to look for interactions with MLPK. MLPK and the kinase domains of $\mathrm{SRK}_{8}$ and $\mathrm{SRK}_{9}$ were subcloned into pGBKT7 (a bait vector containing a GAL4 DNA binding domain (BD)) and pGADT7 (a prey vector containing a GAL4 DNA activation domain (AD)), and we monitored their interactions in the combinations shown in Figure 1. If the bait and prey interact, the DNA-BD and AD are brought into proximity, thus activating transcription of reporter genes (ADE2 and HIS3) that support yeast growth on the $\mathrm{SD} /$-Leu/-Trp/-Ade/-His medium. However, except for the positive control, no growth was observed in any of the bait and prey combinations (Figure 1, middle and bottom panels).

The conventional yeast two-hybrid system has long been an effective tool in detecting protein-protein interactions of nuclear and cytoplasmic proteins. However, the interactions of membrane proteins, such as receptor kinases, G-protein-coupled receptors, membrane-bound

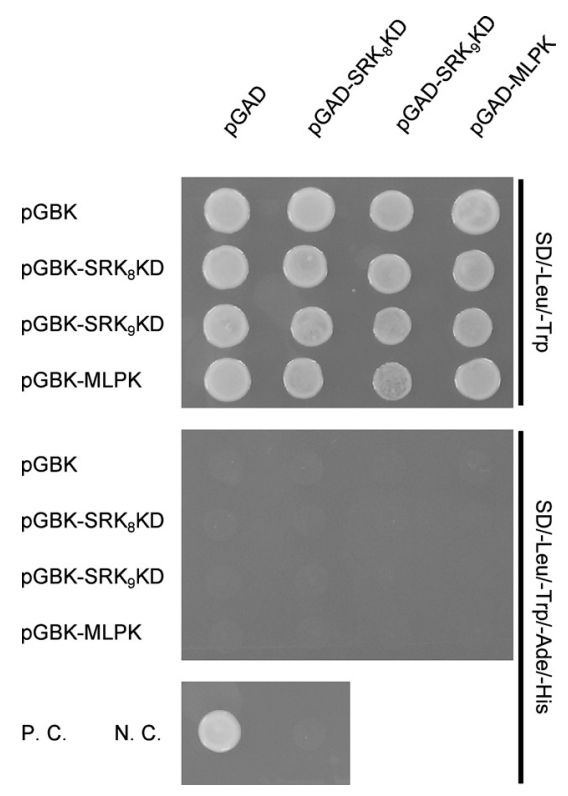

Figure 1. Yeast two-hybrid assay of the interactions between MLPK and the kinase domains of $\mathrm{SRK}_{8}$ and $\mathrm{SRK}_{9}$. Yeast strain $\mathrm{AH} 109$ was co-transformed with the prey and bait vectors as indicated and plated on SD medium lacking Leu and Trp (top panel; SD/-Leu/-Trp) or lacking Leu, Trp, His, and adenine (middle and bottom panels; SD/Leu/-Trp/-Ade/-His). Prey and bait vectors containing SV-40 large Tantigen (pGAD-T) and murine p53 (pGBKT7-53) were used as a positive control (P.C.). pGAD-T and a bait vector containing human lamin C (pGBKT7-Lam) were used as a negative control (N.C.). Growth on the latter medium indicates an interaction between the bait and prey. 
phosphatases, and transporters, have been difficult to detect thus far. To date, the split-ubiquitin membranebased yeast two-hybrid assay system has been considered the most effective means to monitor protein-protein interactions between membrane proteins (Iyer et al. 2005; Stagljar et al. 1998). Thus, we next tried this system to look for a possible interaction between the membrane-anchored forms of SRK and MLPK. The fulllength $\mathrm{SRK}_{8}$ and $\mathrm{SRK}_{9}$ (baits) were cloned into the bait vector (pCCW-SUC) for expression as fusions to the Cterminal half of ubiquitin (Cub) connected with an artificial transcription factor (LexA-VP16). The fulllength MLPK (prey) was cloned into the prey vector (pPR3-C) for expression as a fusion to the mutated $\mathrm{N}$-terminal half of ubiquitin (NubG, which replaces the wild-type isoleucine at position 13 with glycine). Expression and membrane localization of these constructs were confirmed by Western blot, as described in the
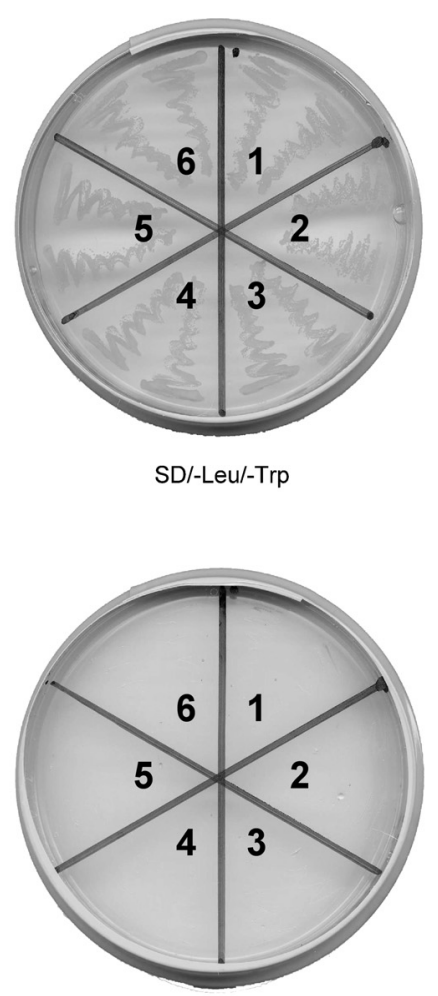

SD/-Leu/-Trp/-Ade/-His

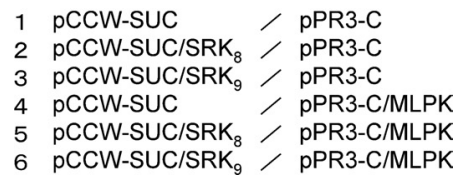

Figure 2. The split-ubiquitin membrane-based yeast two-hybrid assay of the interactions between the SRKs and MLPK. Yeast strain NMY32 was co-transformed with the prey and bait vectors as indicated and plated on SD medium lacking Leu and Trp (top panel; SD/-Leu/-Trp) or lacking Leu, Trp, His, and adenine (bottom panel; SD/-Leu/-Trp/Ade/-His).
Materials and Methods (data not shown). If SRK and MLPK interact, NubG and Cub would be forced into close proximity and result in the reconstruction of the split-ubiquitin. The split-ubiquitin is recognized by ubiquitin-specific proteases that release the artificial transcription factor (LexA-VP16) and activate a set of reporter genes, allowing yeast growth on the SD/-Leu/Trp/-Ade/-His medium. However, as shown in Figure 2, no such interaction was observed for either $\mathrm{SRK}_{8}$-MLPK or $\mathrm{SRK}_{9}-\mathrm{MLPK}$. These results suggested that the interaction between SRK and MLPK, if present, is relatively weak or transient.

Even though our data thus far did not suggest a stable interacting complex between SRK and MLPK, the possibility remained that SI signaling might induce a transient SRK-MLPK interaction that results in the inter-molecular phosphorylation of MLPK. Thus, we next tried to indirectly detect a transient interaction using an in vitro phosphorylation assay. To examine if MLPK could be a substrate for SRK kinase, E. coliexpressed recombinant MLPK protein was incubated with the similarly produced recombinant SRK kinase domain in the presence of $\left[\gamma_{-}{ }^{32} \mathrm{P}\right]$-ATP (Figure 3). In this in vitro phosphorylation assay, we used as a substrate the kinase-deficient form of $\mathrm{MLPK}^{\mathrm{K} 112 \mathrm{R}}$, in which the lysine residue at position 112 (the deduced ATP-binding site) was substituted to arginine, because the wildtype MLPK exhibited very strong autophosphorylation activity, which made it difficult to detect cross intermolecular phosphorylation by SRK (data not shown).

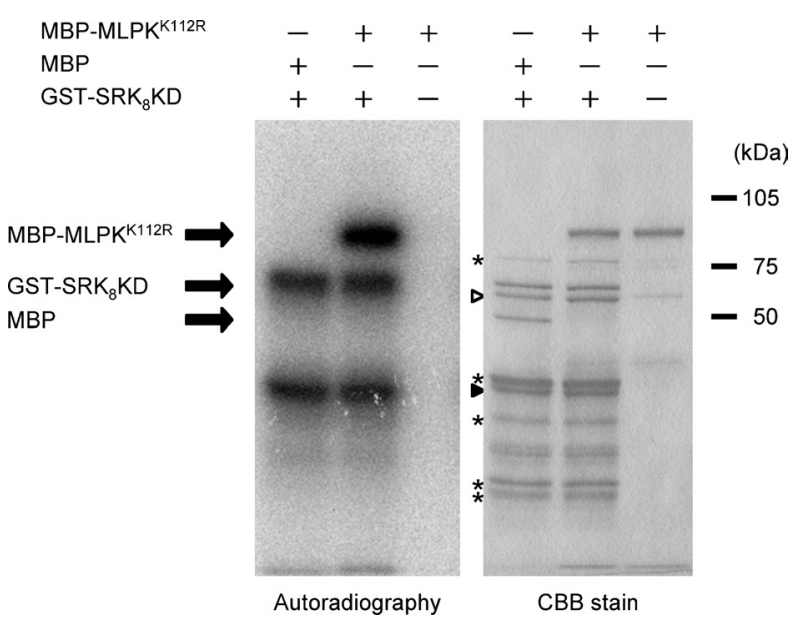

Figure 3. In vitro phosphorylation assay. MBP fusions of kinasedeficient MLPK protein (MBP-MLPK ${ }^{\mathrm{K} 112 \mathrm{R}}$ ) and MBP (control) protein were incubated with recombinant $\mathrm{SRK}_{8}$ kinase domain $\left(\mathrm{GST}-\mathrm{SRK}_{8} \mathrm{KD}\right.$ ) in the presence of $\left[\gamma_{-}{ }^{32} \mathrm{P}\right]-\mathrm{ATP}$. The reaction mixtures were separated on a $10 \%$ SDS-PAGE gel and detected by Coomassie brilliant blue staining (right panel) and autoradiography (left panel). Asterisks indicate the contaminants in the recombinant proteins. The open arrowhead indicates the contaminated E. coli GroEL protein, and the closed arrowhead indicates the degradation product of GST-SRK ${ }_{8} \mathrm{KD}$. The identification of these preoteins was done by LC-MS/MS analysis as described previously (Shimosato et al. 2007). 
The kinase deficient MLPK $^{\mathrm{K} 112 \mathrm{R}}$ mutant exhibited no autophosphorylation activity (Figure 3, right lane), but the GST fusion of SRK kinase domain exhibited autophosphorylation activity and exhibited a radioactive band at ca. $65 \mathrm{kDa}$. Notably, the SRK kinase domain did not phosphorylate MBP protein (control) nor other contaminating proteins, such as GroEL (Figure 3, left lane, ca. $70 \mathrm{kDa}$ ). In contrast, when the SRK kinase domain was incubated with the MBP fusion protein of MLPK $^{\mathrm{K} 112 \mathrm{R}}$, MBP-MLPK ${ }^{\mathrm{K} 112 \mathrm{R}}$ was efficiently phosphorylated (Figure 3, middle lane). These results suggested that MLPK could be a direct and efficient substrate for SRK kinase.

\section{Discussion}

In the present study, we used two different types of yeast two-hybrid systems to look for a possible interaction between SRK and MLPK, but failed to detect any direct interaction. On the other hand, our in vitro phosphorylation assay suggested that MLPK could act as an efficient substrate for the SRK kinase. Taken together, the failure to detect a direct SRK-MLPK interaction could be due to their transient interaction, as observed in certain interactions between mammalian kinases and their substrates (Miyazawa et al. 2002; Waas and Dalby 2002). However, the split-ubiquitin system that we used has been considered suitable for detecting transient interactions (Stagljar et al. 1998). Thus, we must consider other possibilities, e.g. that the interaction between SRK and MLPK might be autoinhibited in the absence of SP11 ligand on the plasma membrane, as has recently been suggested in the case of the interaction between brassinosteroid receptor BRI1 and its coreceptor BAK1 (Belkhadir and Chory 2006).

Although we have shown in this study that MLPK is an efficient substrate for SRK kinase in vitro, it is unclear whether this reflects the situation in vivo. The next goal of this study, therefore, is to detect the SRK-MLPK interaction in vivo. To achieve this goal, recently developed non-invasive fluorophorebased protein interaction assays, such as fluorescence resonance energy transfer (FRET) and bimolecular florescence complementation (BiFC), are expected to be useful (Bhat et al. 2006). To this end, we have already succeeded in transiently expressing a green fluorescent protein (GFP) fusion of MLPK in tobacco BY-2 cell membranes (Murase et al. 2004). We have also succeeded in expressing intact SRK and reconstituting the active (high-affinity SP11 binding) receptor on tobacco BY-2 cell membranes (Shimosato et al. 2007). However, despite much effort, we have not yet succeeded in expressing the GFP fusion protein of SRK, probably due to its large molecular size. Efforts are ongoing to elucidate the physiological relationship between SRK and MLPK in Brassica SI signaling.

\section{Acknowledgments}

We thank Aya Ohta, Hitomi Ichikawa, Maki Matsumura, Yoshie Ohnishi, and Humiyo Yamamoto for their technical assistance. This work was supported by Grants-in-Aid for Scientific Research (Nos. 16GS0316, 16380072, 18380069, and 18075008), and by the 21 st Century Center of Excellence (COE) Program to Nara Institute of Science and Technology from the Ministry of Education, Culture, Sports, Science and Technology of Japan (MEXT).

\section{References}

Belkhadir Y, Chory J (2006) Brassinosteroid signaling: a paradigm for steroid hormone signaling from the cell surface. Science 314: 1210-1411

Bhat RA, Lahaye T, Panstruga R (2006) The visible touch: in planta visualization of protein-protein interactions by gluorephore-based methods. Plant Methods 2: 12

Boisson B, Giglione C, Meinnel T (2003) Unexpected protein families including cell defense components feature in the $\mathrm{N}$ myristoylome of a higher eukaryote. J Biol Chem 278: 4341843429

Cabrillac D, Cock JM, Dumas C, Gaude T (2001) The S-locus receptor kinase is inhibited by thioredoxins and activated by pollen coat proteins. Nature 410: 220-223

Iyer K, Burkle L, Auerbach D, Thaminy S, Dinkel M, Engels K, Stagliar I (2005) Utilizing the split-ubiquitin membrane yeast two-hybrid system to identify protein-protein interactions of integral membrane proteins. SCi STKE 2005 (275): p13

Miyazawa K, Shinozaki M, Hara T, Furuya T, Miyazono K (2002) Two major Smad pathways in TGF- $\beta$ superfamily signalling. Genes Cells 7: 1191-1204

Murase K, Shiba H, Iwano M, Che F-S, Watanabe M, Isogai A, Takayama S (2004) A membrane-anchored protein kinase involved in Brassica self-incompatibility signaling. Science 303: $1516-1519$

Nou IS, Watanabe M, Isogai A, Hinata K (1993) Comparison of $S$ alleles and S-glycoproteins between two wild populations of Brassica campestris in Turkey and Japan. Sex Plant Reprod 6: 79-86

Schopfer CR, Nasrallah ME, Nasrallah JB (1999) The male determinant of self-incompatibility in Brassica. Science 286: $1697-1700$

Shiba H, Takayama S, Iwano M, Shimosato H, Funato M, Nakagawa T, Che F-S, Suzuki G, Watanabe M, Hinata K, Isogai A (2001) A pollen coat protein, SP11/SCR, determines the pollen $S$-specificity in the self-incompatibility of Brassica species. Plant Physiol 125: 2095-2103

Shimosato H, Yokota N, Shiba H, Iwano M, Entani T, Che F-S, Watanabe M, Isogai A, Takayama S (2007) Characterization of the SP11/SCR high-affinity binding site involved in self/nonself recognition in Brassica self-incompatibility. Plant Cell (in press)

Shiu SH, Bleecker AB (2001) Receptor-like kinase from Arabidopsis form a monophyletic gene family related to animal receptor kinases. Proc Natl Acad Sci USA 98: 10763-10768

Stagljar I, Korostensky C, Johnsson N, te Heesen S (1998) A genetic system based on split-ubiquitin for the analysis of 
interactions between membrane proteins in vivo. Proc Natl Acad Sci USA 95: 5187-5192

Stein JC, Howlett B, Boyes DC, Nasrallah ME, Nasrallah JB (1991) Molecular cloning of a putative receptor protein kinase gene encoded at the self-incompatibility locus of Brassica oleracea. Proc Natl Acad Sci USA 88: 8816-8820

Stone SL, Anderson EM, Mullen RT, Goring DR (2003) ARC1 is an E3 ubiquitin ligase and promotes the ubiquitination of proteins during the rejection of self-incompatible Brassica pollen. Plant Cell 15: 885-898

Stone SL, Arnoldo M, Goring DR (1999) A breakdown of Brassica self-incompatibility in ARC1 antisense transgenic plants. Science 286: 1729-1731

Suzuki G, Kai N, Hirose T, Fukui K, Nishio T, Takayama S, Isogai A, Watanabe M, Hinata K (1999) Genomic organization of the $S$ locus: identification and characterization of genes in SLG/SRK region of $S_{9}$-haplotype of Brassica campestris (syn. rapa). Genetics 153: 391-400
Takasaki T, Hatakeyama K, Suzuki G, Watanabe M, Isogai A, Hinata K (2000) The $S$ receptor kinase determines selfincompatibility in Brassica stigma. Nature 403: 913-916

Takayama S, Isogai A (2005) Self-incompatibility in plants. Annu Rev Plant Biol 56: 467-489

Takayama S, Shiba H, Iwano M, Shimosato H, Che F-S, Kai N, Watanabe M, Suzuki G, Hinata K, Isogai A (2000) The pollen determinant of self-incompatibility in Brassica campestris. Proc Natl Acad Sci USA 97: 1920-1925

Takayama S, Shimosato H, Shiba H, Funato M, Che F-S, Watanabe M, Iwano M, Isogai A (2001) Direct ligand-receptor complex interaction controls Brassica self-incompatibility. Nature 413: 534-538

Waas WF, Dalby KN (2002) Transient protein-protein interactions and a random-ordered kinetic mechanism for the phosphorylation of a transcription factor by extracellular-regulated protein kinase 2. J Biol Chem 277: 12532-12540 